\title{
TITLE:
}

\section{Thermoreversible gelation is a Bose-Einstein condensation}

\author{
AUTHOR(S): \\ Tanaka, $\mathrm{F}$
}

\section{CITATION:}

Tanaka, F. Thermoreversible gelation is a Bose-Einstein condensation. PHYSICAL REVIEW E 2006, 73(6): 061405.

\section{ISSUE DATE:}

2006-06

URL:

http://hdl.handle.net/2433/39915

RIGHT:

Copyright 2006 American Physical Society 
PHYSICAL REVIEW E 73, 061405 (2006)

\title{
Thermoreversible gelation is a Bose-Einstein condensation
}

\author{
Fumihiko Tanaka* \\ Department of Polymer Chemistry, Graduate School of Engineering, Kyoto University, Katsura, Kyoto 615-8510, Japan
}

(Received 23 July 2005; revised manuscript received 27 December 2005; published 13 June 2006)

\begin{abstract}
On the basis of the classical tree statistics, being combined with the lattice theory of a polymer solution, we prove that within the mean-field approximation thermoreversible gelation of polyfunctional molecules is a third order phase transition analogous to the Bose-Einstein condensation of ideal Bose gases. The derivative of the osmotic compressibility with respect to the concentration of primary functional molecules is shown to reveal a discontinuity at the sol/gel transition point, whose width is directly related to the amplitude of the divergent term in the weight-average molecular weight of clusters. An inclusion of cycle formation, as well as concentration fluctuations, may, however, change the nature of the phase transition.
\end{abstract}

DOI: 10.1103/PhysRevE.73.061405

PACS number(s): 82.70.Gg, 64.60.-i, 64.70.-p, 64.75.+g

\section{INTRODUCTION}

Recently, thermoreversible gelation in solutions of polymers, as well as low molecular weight molecules, has been attracting researchers' interest [1,2]. Typical examples of the phase diagrams with sol/gel transition lines have been reported in the literature. These include atactic polystyrene in various organic solvents $[3,4]$, poly $(n$-butylmethacrylate) in 2-propanol [5,6], poly(vinyl chloride) in dimethyl malonate [7], etc. Many other examples can be found in the reviews $[1,2]$. However, the thermodynamic nature of the transition from a sol state to a gel state, and vice versa (sol/gel transition), has not yet been clarified. Hence, there have been intense argument and serious confusion on whether or not the thermoreversible gelation is a real phase transition that is accompanied by a singularity in the physical properties. One group of researchers [8] insists that gelation is related only to the connectivity properties of the system, and hence should be a continuous change. Their argument is based on a simple assumption such that gelation is a percolation, and since a percolation is a geometric transition with no thermodynamic singularity associated, sol/gel transition should not have any singularity. Another extremity [9] insists that the transition becomes a first order phase transition if the formation of excessive large-scale cycles in the gel part is taken into consideration. Therefore, it seems to be an urgent issue to resolve this problem.

In our previous study $[10,11]$, we showed that the transition is a third order phase transition analogous to the BoseEinstein condensation (referred to as BEC) of ideal Bose gases. It is well known that an ideal Bose-Einstein gas realizes a characteristic state of order when approarching to the absolute zero temperature [12]. The transition is accompanied by a thermal discontinuity. At a finite temperature given in terms of the number density of Bose molecules, the specific heat at a constant volume has a peak with discontinuous slope. Below this transition temperature, a finite fraction of all molecules goes into the lowest state with zero momentum. The process is characterized as a gradual condensation in momentum space from one phase with finite momentum

*Electronic address: ftanaka@phys.polym.kyoto-u.ac.jp into the condensed phase with zero momentum, although there is no phase separation in space. Our argument $[10,11]$ was based on the fact such that each connected cluster made up of the primary molecules in the solution has a center of mass translational degree of freedom (momentum) contributing to the entropy of mixing. Clusters of finite size (sol) have finite momenta. At the gel point, the largest cluster grows to a macroscopic network (gel). Since the network spans the entire solution, its center of mass ceases translational motion, thus leading to a vanishing of the mixing entropy originating in this cluster.

The gel point to be studied here is defined, in accordance with the classical gelation theory [13-15], by the point where the weight-average molecular weight of the cluster becomes infinite. In other words, it is defined by the percolation point where a connected cluster grows to the macroscopic dimensions to percolate over the entire solution. Such a definition of the gel point on the basis of the connectivity of the system is, in principle, different from the rheological gel point that is defined by the point where the solution loses its fluidity. For chemical gels with covalent cross links, these two definitions give the same point, but we do not study chemical gels here because they are thermodynamically irreversible. In what follows we will focus on physical gels with cross links that may break and recombine to reach thermal equilibrium. Although solutions are percolated by the gel networks, they may flow by repeating association-dissociation of the cross links when external stress is given. Such fluidity of the system does not affect the nature of the Bose-Einstein singularity, although the amplitude of the singularity depends upon the strength of the cross-link bonds described by the association constant $\lambda(T)$ to be studied below.

In the previous study, we showed $[10,11]$ that the second order derivative of the mixing entropy (third order derivative of the free energy) with respect to the temperature or concentration exhibits a discontinuity under the mean-field assumptions such that (i) the free energy of clusters in the solution is given by the Flory-Huggins theory of polymer solutions, (ii) the clusters take Cayley tree forms, and (iii) reactivity of the functional groups is not affected by the full effect of self-avoidance on the lattice. The calculation for proving this, however, involved complicate combinatorial consideration since we treated multiple associations. There- 
fore, the paper seemed to be difficult to access for the majority of researchers. In what follows, we attempt to draw the same conclusion more clearly on the basis of a simpler pairwise cross linking, and stress the analogy between thermoreversible gelation and BEC. Since our proof of the parallel to BEC is based on the above mean-field assumptions (i)-(iii), it may fail when any of these assumptions is eliminated.

\section{THEORETICAL MODEL OF GELLING POLYMER SOLUTIONS}

We start from a model solution in which primary molecules of the molecular weight $n$ (in terms of the number of statistical units on them) carrying the number $f$ of functional groups are dissolved in a solvent [16]. For simplicity, we assume that the functionality of the primary molecules is monodisperse and the functional groups form pairwise bonds that can break and recombine during thermal processes. We employ a lattice theoretical description of the Flory-Huggins type [17-19], and incorporate the cluster formation by using the conventional tree statistics of the gelation reaction [13-15]. Our starting free energy is given by

$$
\begin{aligned}
\beta \Delta F= & \sum_{l \geqslant 1} N_{l} \ln \phi_{l}+N_{0} \ln (1-\phi)+\chi \phi(1-\phi) \Omega+\sum_{l \geqslant 1} \Delta_{l} N_{l} \\
& +\delta(\phi) N^{G},
\end{aligned}
$$

where $N_{l}$ is the number of clusters formed by $l$ primary molecules ( $l$-clusters), $\phi_{l}$ their volume fraction, $N_{0}$ the number of solvent molecules, $\phi$ the total volume fraction of the primary molecules, $\Omega$ the total number of the lattice cells, $\chi$ is Flory's $\chi$ parameter, and $\Delta_{l} \equiv \beta\left(\mu_{l}^{\circ}-l \mu_{1}^{\circ}\right)$, the free energy change accompanying the formation of an $l$-cluster from the separate primary molecules in their standard reference state (superscript circle). The last term is necessary only in the postgel regime where a gel network formed by a macroscopic number $N^{G}$ of primary molecules exists. The free energy to bind a molecule onto the gel is given by $\delta(\phi) \equiv \beta\left(\mu^{\circ G}-\mu_{1}^{\circ}\right)$. By differentiation, we find for chemical potentials

$$
\begin{aligned}
\beta \Delta \mu_{l}= & \Delta_{l}+1+\ln \phi_{l}-n l \rho+\chi n l(1-\phi)^{2} \\
& +n l \delta^{\prime}(\phi) \nu^{G}(1-\phi), \\
\beta \Delta \mu_{0}=1 & +\ln (1-\phi)-\rho+\chi \phi^{2}-\delta^{\prime}(\phi) \nu^{G} \phi,
\end{aligned}
$$

for the $l$ cluster and the solvent molecule, where

$$
\rho \equiv \sum_{l \geqslant 1} \nu_{l}+1-\phi
$$

gives the total number of molecules and clusters possessing the translational degree of freedom. The summation

$$
\nu \equiv \sum_{l \geqslant 1} \nu_{l}
$$

gives the number density of finite clusters in the solution, and $\nu^{G} \equiv N^{G} / \Omega$ is the number density of polymer chains contained in the gel network.

In thermal equilibrium, the solution has a distribution of clusters with a population distribution fixed by the equilibrium condition

$$
\Delta \mu_{l}=l \Delta \mu_{1}
$$

for association and dissociation. Then, we find the volume fraction of $l$ clusters to be given by

$$
\phi_{l}=K_{l} \phi_{1}{ }^{l},
$$

where $\phi_{1}$ is the volume fraction of the unassociated molecules, and the equilibrium constant is given by $K_{l}$ $=\exp \left(l-1-\Delta_{l}\right)$.

\section{APPLICATION OF THE CLASSICAL TREE STATISTICS FOR GELATION}

We now split the free energy into three parts $\Delta_{l}=\Delta_{l}^{\text {comb }}$ $+\Delta_{l}^{\text {conf }}+\Delta_{l}^{\text {bond }}$. To find the combinatorial part, all clusters are assumed to take tree forms. The cycle formation within a cluster is neglected. We consider the entropy change on combining $l$ identical $f$-functional molecules to form a single Cayley tree. The classical tree statistics [14] gives $\Delta S_{l}^{\text {comb }}$ $=k_{B} \ln \left[f^{l} \omega_{l}\right]$, where

$$
\omega_{l} \equiv \frac{(f l-l) !}{l !(f l-2 l+2) !}
$$

is Stockmayer's combinatorial factor. The free energy is given by $\Delta_{l}^{\text {comb }}=-\Delta S_{l}^{\text {comb }} / k_{B}$.

For the conformational free energy, we employ the lattice theoretical entropy of disorientation [19],

$$
S_{\text {dis }}(n)=k_{B} \ln \left(\frac{n \zeta(\zeta-1)^{n-2}}{\sigma e^{n-1}}\right)
$$

for a chain consisting of $n$ statistical units, where $\zeta$ is the lattice coordination number, and $\sigma$ the symmetry number of the chain. We then find

$$
\Delta S_{l}^{\mathrm{conf}}=S_{\mathrm{dis}}(l n)-l S_{\mathrm{dis}}(n)=k_{B} \ln \left[\left(\frac{\sigma(\zeta-1)^{2}}{\zeta e n}\right)^{l-1} l\right],
$$

with most probably $\sigma=1$.

Finally, the free energy of bonding is given by

$$
\Delta_{l}^{\text {bond }}=(l-1) \beta \Delta f_{0},
$$

because there are $l-1$ bonds in a tree of $l$ molecules, where $\Delta f_{0}$ is the free energy change on forming one bond.

Combining all results together, we find

$$
K_{l}=f l \omega_{l}\left(\frac{f \lambda}{n}\right)^{l-1}
$$

for the equilibrium constant, where

$$
\lambda(T) \equiv\left[\sigma(\zeta-1)^{2} / \zeta e\right] \exp \left(-\beta \Delta f_{0}\right)
$$

is the association constant.

We first consider the pregel regime where all clusters are finite. The total volume fraction and the total number of clusters in the solution are then given by using Eq. (2.6) as

$$
\lambda \phi / n=\sum_{l=1}^{\infty} l \omega_{l} x^{l},
$$




$$
\lambda \nu=\sum_{l=1}^{\infty} \omega_{l} x^{l}
$$

Here, the parameter $x$ is defined by $x \equiv \lambda \phi_{1} / n$ ( $\phi_{1}$ being the volume fraction of primary molecules that remain unassociated in the solution). The osmotic pressure $\pi$ of the solution is given by the chemical potential of the solvent through the thermodynamic relation $\pi a^{3}=-\Delta \mu_{0}$. Explicitly, we have

$$
\pi a^{3} / k_{B} T=\nu-\phi-\ln (1-\phi)-\chi \phi^{2}
$$

in the pregel regime. It is basically proportional to the total number $\nu$ of clusters since all molecules with a translational degree of freedom equally contribute to the pressure within the ideal gas approximation. By solving Eq. (3.7a) with respect to $x$, and substituting the result into Eq. (3.8), we obtain the osmotic pressure as a function of the temperature and volume fraction of the primary molecules.

\section{SIMILARITY TO BOSE-EINSTEIN CONDENSATION}

At this stage, we readily realize that our equations are mathematically parallel to those we encounter in the study of BEC of ideal Bose gases [12,20]. The number density $N / V$ and the pressure $p$ of an ideal Bose gas consisting of $N$ molecules confined in the volume $V$ is given by

$$
\begin{gathered}
\lambda_{T}^{3} N / V=\sum_{l=1}^{\infty} x^{l} / l^{3 / 2}, \\
p \lambda_{T}^{3} / k_{B} T=\sum_{l=1}^{\infty} x^{l} / l^{5 / 2},
\end{gathered}
$$

where $x$ is the activity of the molecule, and $\lambda_{T}$ $\equiv h /\left(2 \pi m k_{B} T\right)^{1 / 2}$ the thermal de Broglie wavelength. The coefficient of the infinite series on the right-hand side is replaced from Stockmayer's combinatorial factor $\omega_{l}$ to $1 / l^{5 / 2}$, but other parts are completely analogous. The infinite summations on the right-hand side of these equations are known as Truesdell's function [21] of order $\frac{3}{2}$ and $\frac{5}{2}$. Their singularity appearing at the convergence radius $x=1$ was studied in detail [21]. Since the internal energy of the Bose gas is related to the pressure as $U=3 p V / 2$, the singularity in the compressibility and that in the specific heat has the same nature and reveal discontinuity in their derivatives [12]. Hence the transition (condensation of macroscopic number of molecules into a single quantum state) turns out to be a third order phase transition [12,20,22].

We now show that a similar picture holds for our gelling solution; a finite fraction of the total number of primary molecules condenses into a single state (gel network) with no center of mass translational degree of freedom (no momentum), although we have no quantum effect. We first calculate the (dimensionless) osmotic pressure defined by $K_{T}$ $\equiv\left(k_{B} T / a^{3}\right)(\partial \pi / \partial \phi)_{T} / \phi$ as a function of the temperature and the volume fraction. By taking the concentration derivative of Eq. (3.8), we find $K_{T}^{-1}=\phi^{2} \sigma(\phi, T)$, where

$$
\sigma(\phi, T) \equiv \kappa(\phi, T) / n \phi+1 /(1-\phi)-2 \chi
$$

Here, a new function $\kappa$ is defined by $\kappa \equiv n(\partial \nu / \partial \phi)_{T}$. This result was previously derived in the case of multiple cross links [10]. The singularity in the osmotic pressure originates in this $\kappa$ function: the translational entropy of clusters. From the fundamental two relations (3.7a) and (3.7b) given above, the function $\kappa$ is given by

$$
\kappa=\sum l \omega_{l} x^{l} / \sum l^{2} \omega_{l} x^{l}=1 / \bar{l}_{\mathrm{w}}
$$

in terms of the weight-average aggregation number $\bar{l}_{\mathrm{w}}$ of clusters. In what follows, we show that $\kappa$ is continuous across the gel point concentration, but its derivative $(\partial \kappa / \partial \phi)_{T}$ reveals a discontinuity.

To simplify theoretical analysis, let us introduce the $k$ th moment of Stockmayer's distribution by

$$
S_{k}(x) \equiv \sum_{l=1}^{\infty} l^{k} \omega_{l} x^{l} \quad(k=0,1,2, \ldots) .
$$

Then, the number- and weight-average cluster sizes are given by

$$
\begin{aligned}
& \bar{l}_{\mathrm{n}}=S_{1}(x) / S_{0}(x), \\
& \bar{l}_{\mathrm{w}}=S_{2}(x) / S_{1}(x) .
\end{aligned}
$$

All moments are monotonically increasing functions of $x$ and have a common radius of convergence

$$
x=x^{*} \equiv(f-2)^{f-2} /(f-1)^{f-1} .
$$

For $x>x^{*}$ all moments diverge. Exactly on the radius of convergence $x^{*}, S_{0}$ and $S_{1}$ take finite values, but all moments with $k \geqslant 2$ are infinite. Since the weight-average cluster size becomes infinite at this point, $x=x^{*}$ gives the gel point. The volume fraction at the gel point is therefore given by $\lambda \phi^{*} / n=S_{1}\left(x^{*}\right)=(f-1) / f(f-2)^{2}$. With further increase in the volume fraction of primary molecules, the excess molecules in the part $\phi-\phi^{*}$ condense into the macroscopic cluster (infinitely extended branched network) through a cascade process, and will not contribute to the number density, so that it will remain a constant value $\lambda \nu^{*}=2(f-1) /(f-2)$. The weight-average cluster size also remains infinite. We have, therefore, a kind of condensation phenomenon in momentum space.

The analogy of BEC can be seen more clearly if we replace Stockmayer's combinatorial factor $\omega_{l}$ by its asymptotic form $\omega_{l} \simeq x^{*-1} / l^{5 / 2}$ for large $l$. This form is derived by applying Stirling's formula to Eq. (3.1). We find

$$
\begin{gathered}
\lambda \phi / n=\sum_{l=1}^{\infty} \frac{1}{l^{3 / 2}}\left(\frac{x}{x^{*}}\right)^{l}, \\
\lambda \nu=\sum_{l=1}^{\infty} \frac{1}{l^{5 / 2}}\left(\frac{x}{x^{*}}\right)^{l} .
\end{gathered}
$$

Thus, we can see that the singularity at $x=x^{*}$ is perfectly identical to those in Truesdell's functions at $x=1$. In our 
previous study [10], we referred to this important similarity in the case of multiple association.

A simple calculation gives

$$
\bar{l}_{\mathrm{w}} \simeq A /\left(\phi^{*}-\phi\right)
$$

with a constant amplitude

$$
A \equiv f n /(f-2)^{3} \lambda(T)
$$

for $\phi<\phi^{*}$, and $\bar{l}_{\mathrm{w}}=\infty$ for $\phi>\phi^{*}$. We thus find the discontinuity in the slope of the function $\kappa$ is given by $\Delta(\partial \kappa / \partial \phi)_{T}$ $=1 / A$. This leads to a discontinuity in the osmotic compressibility of the form

$$
\Delta\left(\frac{\partial K_{T}}{\partial \phi}\right)_{T}=-K_{T}^{2}\left(\frac{\phi^{*}}{n}\right) \Delta\left(\frac{\partial \kappa}{\partial \phi}\right)_{T}=-B / \sigma\left(\phi^{*}, T\right)^{2},
$$

where

$$
B \equiv f^{2}(f-2)^{9} \lambda(T)^{4} /(f-1)^{3} n^{5}
$$

is a constant depending only on the temperature, functionality, and the number of statistical units on a chain. For large molecular weight polymers, the amplitude $B$ is small. This is the main reason why an experimental detection of the singularity has so far been difficult. However, there is a good chance for the observation to be feasible. Quite often, the sol/gel transition line crosses the phase separation line (binodal and spinodal) at the top, or on the shoulder, of the phase separation region. Such a crossing point is called either a tricritical point or critical endpoint depending upon the relative position of the transition curves. As we approach the spinodal point (by changing the temperature under a fixed concentration, for instance) where the condition $\sigma\left(\phi^{*}, T\right)$ $=0$ is satisfied, the discontinuity is enhanced by critical fluctuations as seen from Eq. (4.10), and there may be a chance to observe the singularity.

\section{BOSE-EINSTEIN CONDENSATION IN CLASSICAL STATISTICAL MECHANICS}

There are several other condensation phenomena in classical statistical mechanics that are similar to BEC. Jacobson and Stockmayer [23], for example, pointed out that, for linear polycondensation systems with chains and rings, it is possible to obtain $100 \%$ yield of rings beyond a certain critical dilution by driving the polycondensation to completion. The origin of this singularity comes from the entropy of rings. They showed that, within Gaussian chain statistics, the population of rings of size $l$ is proportional to $x^{l} / l^{5 / 2}$, where $x$ is the molar concentration of the primary molecules that remain unassociated in the solution. Similar singularity, and occurrence of a phase transition, was reported in the study of the melting of nucleic acids [24]. In the melting of duplex DNA, the entropy of loops formed by the complementary chains due to partial dissociation of the hydrogen bonds gives rise to a singularity. The nature of the phase transition depends upon the index $c$ characterizing the loop entropy in the form $1 / l^{c}$ for a large number of monomer units $l$. It was pointed out that the case $c=\frac{5}{2}$ turns out to be analogous to BEC. The Stockmayer factor in the combinatorial tree statistics falls also on this category. The effect of cycle formation in the sol, as well as in the gel, beyond the level of tree statistics, is however important. It is well known that the gel point shifts to a higher conversion, and modifies the critical index of divergence in $\bar{l}_{\mathrm{w}}$. It may also modify the nature of the phase transition as was discussed in Ref. [9], although their argument was not plausible. (In the case of the first order transition, there should be a finite concentration gap between the sol phase and gel phase, called "chimney," on the temperature-concentration phase plane that comes out of the macroscopic phase separation region, but this has never been observed.) What we presented here is a proof for the existence of a third order singularity in thermoreversible gels within the conventional mean-field description of gelation.

\section{TREATMENT OF THE POSTGEL REGIME}

The theoretical argument given here is based on Stockmayer's picture [14] for treating the gelling reaction in the regime after passing the gel point. Theoretically, this is not the only consistent way to treat the postgel regime. There are other ways of treating it that fulfill the fundamental thermodynamic laws (Gibbs-Duhem relation). In fact, Flory had proposed a different picture in his old work on a gelation reaction of trifunctional molecules [13]. In his treatment, molecules in the sol part react with those in the gel part with an increase in the concentration, and as a result, formation of cyclic linkages within the gel part is allowed. The weightaverage cluster size $\bar{l}_{\mathrm{w}}$ in the sol goes back to a finite decreasing function of the concentration because large clusters in the sol part are connected into the gel network as reaction proceeds. Upon adopting this treatment, we readily find

$$
\bar{l}_{\mathrm{w}} \simeq A /\left|\phi-\phi^{*}\right|
$$

near the gel concentration, and hence the discontinuity in the slope of $\kappa$ is doubled as $\Delta(\partial \kappa / \partial \phi)_{T}=2 / A$. The third order nature of the phase transition remains the same as in Stockmayer's picture, although the molecular mechanism of condensation and hence the amplitude of the discontinuity are different. These two treatments do not contradict fundamental laws of thermodynamics, and both are equally acceptable. Their comparison and application to phase diagrams were detailed in our preceding paper [11].

To study the postgel regime in more detail, let us introduce a new parameter $\alpha$ through the positive root of the equation

$$
x \equiv \alpha(1-\alpha)^{f-2} .
$$

Then, the first three moments of Stockmayer's distribution are explicitly calculated as [14]

$$
\begin{gathered}
S_{0}(x)=\alpha(1-f \alpha / 2) / f(1-\alpha)^{2}, \\
S_{1}(x)=\alpha / f(1-\alpha)^{2}, \\
S_{2}(x)=\alpha(1+\alpha) / f[1-(f-1) \alpha](1-\alpha)^{2} .
\end{gathered}
$$


To see the physical meaning of $\alpha$, let us calculate the extent of the reaction, i.e., the probability for a randomly chosen functional group to be associated. Since an $l$ cluster includes the total of $f l$ groups, among which $2(l-1)$ are associated, it is given by $2\left[S_{1}(x)-S_{0}(x)\right] / f S_{1}(x)=\alpha$. Thus, it turns out that $\alpha$ introduced by the formal relation (6.2) actually gives the extent of reaction. By using $\alpha$, the average cluster sizes are given by

$$
\begin{gathered}
\bar{l}_{\mathrm{n}}=1 /(1-f \alpha / 2), \\
\bar{l}_{\mathrm{w}}=(1+\alpha) /[1-(f-1) \alpha] .
\end{gathered}
$$

Since the weight-average diverges at $\alpha=\alpha^{*} \equiv 1 /(f-1)$, the extent of the reaction at the gel point is given by $\alpha^{*}$. This value corresponds to the maximum of $x=x^{*}$ in Eq. (6.2). At low concentration with the extent of reaction smaller than the critical value $\alpha^{*}$, Eq. (6.2) gives a unique value of $\alpha$ for a given $x$. On passing the gel point, $\alpha$ becomes larger than $\alpha^{*}$, so that Eq. (6.2) gives another root $\alpha^{\prime}$ that lies below $\alpha^{*}$. Flory [13] adopted this lower root as the extent of reaction in the sol part, while $\alpha$ is regarded as that of the total solution. In the present context of thermoreversible gelation, the first equation $(3.7 \mathrm{a})$ remains valid in the form

$$
\lambda \phi / n=S_{1}(\alpha)
$$

since it gives the total volume fraction. The second one is changed to

$$
\lambda \nu^{S}=S_{0}\left(\alpha^{\prime}\right),
$$

because the number of clusters can be counted only for finite clusters [25]. (Superscript $S$ indicates the sol part.) The volume fraction of the sol part is similarly given by

$$
\lambda \phi^{S} / n=S_{1}\left(\alpha^{\prime}\right),
$$

leading to the sol fraction $w^{S}=S_{1}\left(\alpha^{\prime}\right) / S_{1}(\alpha)$. The function $\kappa$ that appeared in the compressibility now includes the term proportional to

$$
n(\partial \nu / \partial \phi)_{T}=1 / l_{\mathrm{w}}^{S}
$$

where

$$
\bar{l}_{\mathrm{w}}^{S}\left(\alpha^{\prime}\right)=\left(1+\alpha^{\prime}\right) /\left[1-(f-1) \alpha^{\prime}\right]
$$

refers to the weight-average cluster size in the sol part in the postgel regime. Thus, we find a discontinuity in the slope of the osmotic compressibility also in Flory's treatment.

The difference in the above two treatments were later examined from a kinetic point of view. For an irreversible reaction, Ziff and Stell [26] clarified the reaction mechanism (sol-gel interaction) in the two treatments after the gel point is passed. They found that in Stockmayer's treatment reactive groups in the sol do not interact with those in the gel, and the gel grows through a cascade process of the sol into the gel, while in Flory's treatment all functional groups are allowed to react. On the basis of such a kinetic study, they proposed a third model which takes the reaction between sol and gel network into account, while the cycle formation in the gel is forbidden as in Stockmayer's model. This third treatment, however, turned out not to fulfill Gibbs-Duhem relation when applied to thermoreversible gelation [11]. Later, to ensure the equilibrium distribution, additional terms describing reversible reaction (fragmentation) were introduced to the kinetic equation by van Dongen and Ernst [27]. Since their study was limited only to Flory's and Stockmayer's model, the possibility of another new treatment within the classical tree statistics remains unexcluded. From the mathematical analysis given in this paper, however, it is highly probable that a new thermodynamically consistent treatment, even if it exists, leads to the third order singularity lying somewhere between Stockmayer's one and Flory's one.

\section{CONCLUSIONS AND DISCUSSION}

We have shown that, within the mean-field treatment of both gelation reaction and polymer solution, thermoreversible gelation is a third order phase transition analogous to the BEC. The gel network corresponds to a Bose condensate with no translational motion (momentum) as a whole. There is a frequent exchange of molecules between the gel network (with no momentum) and the sol part (with finite momentum), but on thermal average a finite fraction (gel fraction $w^{G}=1-w^{S}$ ) in the number of molecules loses their momenta in the postgel regime.

The effect of fluctuations in cluster formation is important near the transition point as it usually is in many phase transitions. It may change the nature of the transition. In the present gelation problem, however, one has to consider it from two sides. Firstly, the effect of cycle formation during the gelation reaction beyond the classical tree statistics must be considered. Secondly, concentration fluctuations in the polymer solution must be considered beyond the mean-field Flory-Huggins treatment. These two different aspects of fluctuations should be studied from a unified theoretical point of view without violating the fundamental law of thermodynamics. This is an open problem. The critical exponent and the nature of the singularity may be changed by these fluctuation effects at extremely near the transition point. The criterion for the mean-field prediction to be valid (Ginzburg criterion) should also be found in order to characterize nonclassical singularity in the experimeriments. This problem also lies beyond the scope of the present study.

As for the absolute value of the discontinuity in the slope of the osmotic compressibility, closer experimental observation near the spinodal point with enhanced singularity by critical fluctuations of phase separation is necessary since the amplitude $B$ is small for polymeric gels with $n \gg 1$. (For a trifunctional molecules with $f=3$, for example, we have $B$ $=9 \lambda^{4} / 8 n^{5}$.) For this purpose, measurements near the tricritical point, the point where the sol/gel transition line (continuous transition) crosses the binodal (discontinuous first order transition) at the top of the miscibility gap, are suitable. 
[1] J. M. Guenet, Thermoreversible Gelation of Polymers and Biopolymers (Academic Press, Harcourt Brace Jovanovich Pub., New York, 1992).

[2] K. te Nijenhuis, Adv. Polym. Sci. 130, 1 (1997).

[3] H. M. Tan, A. Moet, A. Hiltnet, and E. Baer,Macromolecules, 16, 28 (1983).

[4] R. F. Boyer, E. Baer, and A. Hiltner, Macromolecules 18, 427 (1985).

[5] J. M. Jelich, S. P. Nunes, E. Paul, and B. A. Wolf, Macromolecules 20, 1943 (1987).

[6] T. Schneider, B. A. Wolf, H. Kasten, and F. Kremer, Macromolecules 24, 5391 (1991)

[7] P. H. Mutin and J. M. Guenet, Macromolecules 22, 843 (1989).

[8] A. N. Semenov and M. Rubinstein, Macromolecules 31, 1373 (1998).

[9] I. Erukhimovich, M. V. Thamm, and A. V. Ermoshkin, Macromolecules 34, 5653 (2001).

[10] F. Tanaka and W. H. Stockmayer, Macromolecules 27, 3943 (1994).

[11] M. Ishida and F. Tanaka, Macromolecules 30, 3900 (1997).

[12] F. London, Superfluids (Dover, New York, 1964), Vol. II, Chap. C.

[13] P. J. Flory, J. Am. Chem. Soc. 63, 3091; 3096 (1941).

[14] W. H. Stockmayer, J. Chem. Phys. 11, 45 (1943); 12, 125
(1944).

[15] P. J. Flory, Principles of Polymer Chemistry (Cornell University Press, Ithaca, NY, 1953), Chap. IX.

[16] F. Tanaka, Macromolecules 22, 1988 (1989).

[17] P. J. Flory, J. Chem. Phys. 10, 51 (1942).

[18] M. L. Huggins, J. Phys. Chem. 46, 151 (1942).

[19] P. J. Flory, Principles of Polymer Chemistry (Ref. [15]), Chap. 12.

[20] J. Mayer and M. G. Mayer, Statistical Mechanics (Wiley, New York, 1940), Chap. 16.

[21] C. Truesdell, Ann. Math. 46, 144 (1945).

[22] J. Mayer and M. G. Mayer, Statistical Mechanics (Ref. [20]), Chap. 14.

[23] H. Jacobson and W. H. Stockmayer, J. Chem. Phys. 18, 1600 (1950).

[24] D. Poland and H. A. Scheraga, J. Chem. Phys. 45, 1464 (1966); Theory of Helix-Coil Transitions in Biopolymers (Academic Press, New York, 1970), Secs. 9E and 10D.

[25] The error in Ref. [8] originates in this point. They claimed that they employed Flory's postgel picture. But, in fact, they missed the extent of reaction $\alpha^{\prime}$ of the sol in the postgel regime.

[26] R. M. Ziff and G. Stell, J. Chem. Phys. 73, 3492 (1980).

[27] P. G. van Dongen and M. H. Ernst, J. Stat. Phys. 37, 301 (1984). 